\title{
Simplified computations of spin excitations in high-spin carbon nanoclusters and related systems
}

\author{
A.V.Luzanov \\ STC "Institute for Single Crystals", National Academy of Sciences of \\ Ukraine, 60 Lenin Ave., 61001 Kharkiv, Ukraine
}

Received August 10, 2015

\begin{abstract}
Spin excitation spectra for possible molecule-based magnetic structures are evaluated semiempirically. A simplest spin-flip model with singly-excited configurations (SF-CIS) is used. While only a small amount of electron correlation is captured by the SF-CIS scheme, it turns out to be practically useful tool for computing quasi-magnon spectra in large polyradicals. We study examples of three different structural types (graphene nanoribbon with methylene edges, triangulene and Mataga-type model organic ferromagnet). We demonstrate that these systems show different behavior of the spin excitation spectra and different spin heat capacity temperature dependences. It is also shown that the same SF-CIS technique can be useful for describing high-spin states in nanodiamonds with defects.
\end{abstract}

Keywords: large spin states, molecular magnets, spin-flip models, $\pi$-conjugated polyradicals, nanodiamond.

Полуэмпирическим методом рассчитаны спектры спиновых возбуждений для возможных молекулярных магнитных структур. Использован простейший спин-флип метод SF-CIS, учитывающий однократно возбужденные конфигурации. Хотя с помощью SF-CIS учитывается только малая доля электронной корреляции, метод оказывается практически пригодным для расчета квазимагнонных спектров в больших полирадикалах. Изучены три типа структур (графеновая нанополоска, триангулен и органический ферромагнетик по Матага). Показано, что данные системы демонстрируют различный характер спектра спиновых возбуждений и различное поведение спиновой теплоемкости, показано также, этот метод SF-CIS может быть полезным для описания высокоспиновых состояний наноалмазных структур с дефектами.

Спрощені обчислення спінових збуджень високоспінових вуглецевих нанокластерів та споріднених систем. А.В.Лузанов.

$\mathrm{y}$ напівемпіричний спосіб обчислено спектри спінових збуджень у можливих молекулярних магнітних структурах. Використовано найпростішу спін-фліп модель SF-CIS, що враховує однократно збуджені конфігурації. Хоча за методом SF-CIS обчислюється лише невелика частина електронної кореляції, метод виявляється практично доцільним для розрахунків квазімагнонних спектрів у великих полірадикалах. Вивчено три різні типи структур (графенова нанострічка з метиленовими кінцівками, триангулен та модельний органічний феромагнетик за типом Матага). Показано, що ці системи демонструють відмінний характер спектрів спінових збуджень та різну температурну залежність спінової теплоємності. Встановлено, що цей метод SF-CIS можна застосовувати для опису високоспінових станів у наноалмазних структурах з дефектами. 


\section{Introduction}

Molecule-based magnets is an active area of modern material science, and the main topics of it have been well reviewed in the recent literature [1-4]. A special interest is given to polyradicals and molecular ferromagmetics, and particularly organic ferromagnetics and organic spin clusters [5-9]. The first theory of high-spin states in the conjugated systems, at a simple $\pi$-electron (Huckel) level, was presented long ago in the pivotal paper [10]. Afterwards, several important $\pi$-magnetic structures were designed by Mataga [11] and Ovchinnikov [12].

An essential advance in our understanding of high-spin radical states is achieved when we go beyond the usual one-electron schemes (such as the Huckel one), and take into account electron correlation effects (see, e.g., [13-15]). In particular, the Lieb-Ovchinnikov $[12,16]$ rule usually yields a correct description of the ground state spin in alternant hydrocarbons, that is in the conjugated systems with bipartite structure.

During the last decade, a lot of works were devoted to the study of ferromagnetic and antiferromagnetic states in graphene nanoclusters ([17-22] and many others). Obviously, simplified and crude models are the only available tools to describe such complex polyatomic systems. For example, the UHF (unrestricted Hartree-Fock) and EHF (extended Hartree-Fock) models can be used for organic polyradicals [23, 25]. Nevertheless, one does not forget to verify, at least for less complicated systems, the reliability of the models used. Sometimes, investigations in this field suffer from the lack of such analysis.

Thus, the main purpose of the present paper is to study typical high-spin carbonbased structures, in particular, graphene nanoclusters with radical spins at edges. In our investigation, we employ the so-called spinflip technique which was used for $\pi$-electrons even in [26].

Notice that the spin-flip (SF) method, quite popular now, has a long history, starting with the celebrated Bethe ansatz in Ref. [27] where the one-dimensional antiferromagnetic Heisenberg spin chain was solved exactly. In the Bethe approach the starting state is a fully ordered ferromagnetic state from which the sought antiferromagnetic state is obtained by successive spin flip transformations (see a clear discussion in [28]). The important was Nagaoka's paper [29] where the single spin flip approach is used to suggest a model for the charged ferromagnetic system (for a further development [30,31]).

Seemingly, spin flip transformations (spin-flipping operators) have been first applied in quantum chemistry [26,32] within $\pi$-conjugated system theory. Then ab initio spin-flip methods were independently developed [33-35], producing more and more interesting results in this direction. In our study we will use a rather simple SF version (with a single spin flip) which follows from the general consideration in $[26,36,37]$. This is the so-called SF-CIS, i.e., a spin-flip counterpart of configuration interaction singles (CIS) method. At the non-empirical level, it was firstly described and investigated in [33].

In the present paper we start with checking in detail the validity of the used $\pi$-electron SF-CIS method. This will be done by invoking the results of the $\pi$-electron full configurational interaction $(\pi-\mathrm{FCI})$ computations on small tetraradical hydrocarbons. Then we describe SF-CIS computations for selected high-spin systems. The technical details of the applied method are described in Appendices. In particular, we examine the possibility to employ SF-CIS for modelling high-spin states in relatively small nanodiamond structures with defects.

\section{Spin-flip technique for radical systems}

Let us recall basic equations of the spin-flip technique for SF-CIS states. Following [36], we start with a high-spin Slater (reference) determinant $\left|\Phi_{*}\right\rangle$ with the given numbers, $N_{\alpha}$ and $N_{\beta}$, of spin-up and spin-down orbitals, respectively. Evidently, the quantity

$$
S_{*}=\left(N_{\alpha}-N_{\beta}\right) / 2
$$

determines the spin $z$-projection value of the reference. In practice, determinant $\left|\Phi_{*}\right\rangle$ is taken as a spin-pure state computed within the ROHF (restricted open-shell Hartree-Fock) scheme. Then the total spin value equals to $S_{*}$, and the squared spin of the reference is $S_{*}\left(S_{*}+1\right)$.

In order to obtain a SF-CIS state $\left|\Psi^{\left(S^{*-1}\right)}\right\rangle$, that is the solution with the total spin value $S_{*}$. -1 , we apply the one-electron transformation:

$$
\left|\Psi^{\left(S_{*}-1\right)}\right\rangle=\sum_{1 \leq i \leq N} \tau(i)\left|\Phi_{*}\right\rangle,
$$

where $\tau$ is a one-electron transition density matrix for $\left|\Phi_{*}\right\rangle \rightarrow\left|\Psi^{\left(S^{*}-1\right)}\right\rangle$, and $\tau(i)$ is the conventional notation for $\tau$ acting on the 
Table 1. Lowest quintet-triplet excitation energy $\lambda[Q \rightarrow T]$ (in $\mathrm{eV}$ ) and spin density distribution for the ground quintet state in small conjugated tetraradicals within $\pi$-FCI.

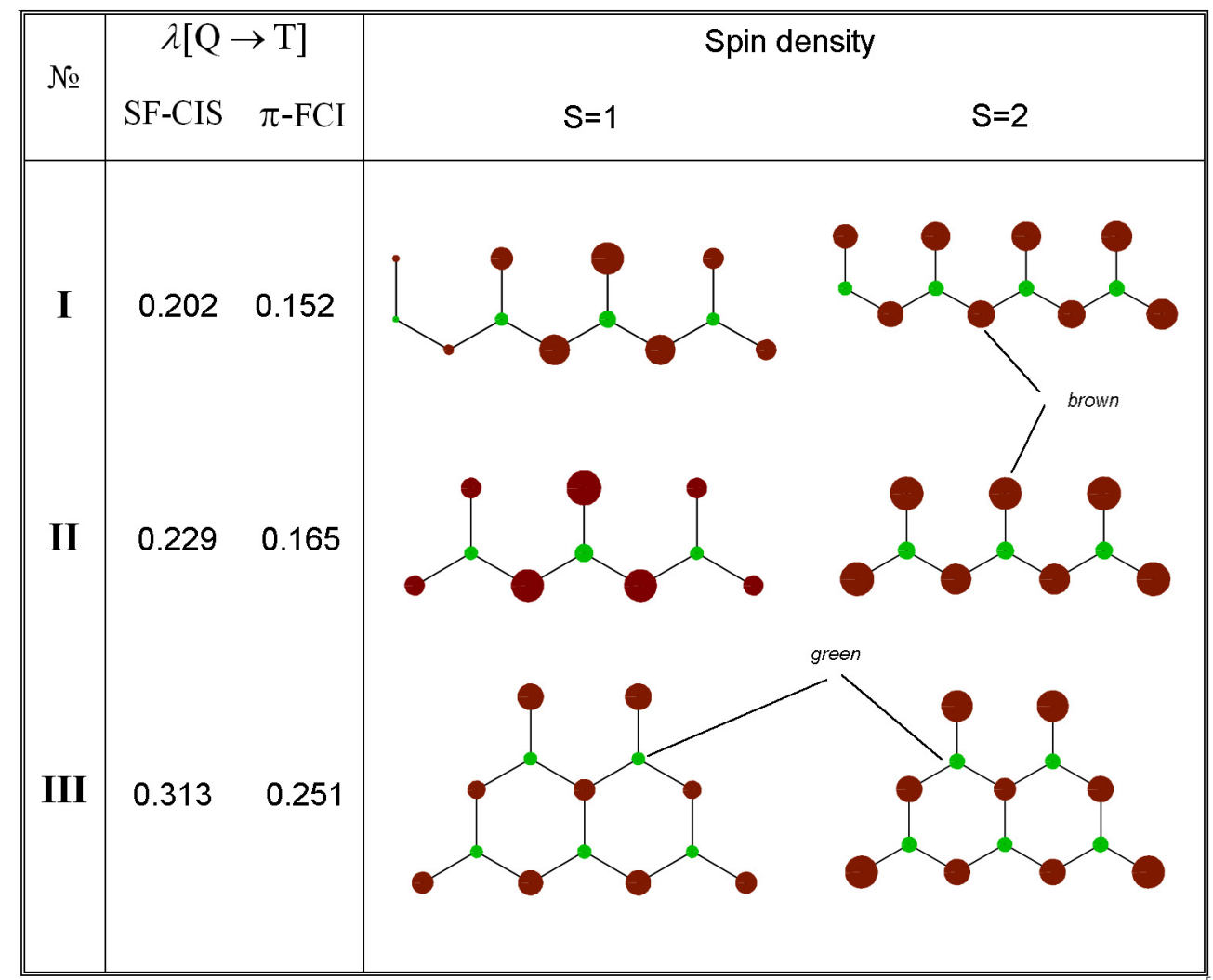

$i$-th electron. A simple spin-flip structure of the $\tau$-operator, in the form

$$
\tau=t s_{-} \equiv t|\beta\rangle\langle\alpha|
$$

with $t$ being a spin-free one-electron amplitude matrix, guarantees a spin projection of state (2) to be $S_{*}-1$. Recall that $|\alpha\rangle$ and $|\beta\rangle$ are the standard spin-up and spin-down eigenkets, respectively.

In what follows, we will use the conventional Pariser-Parr-Pople (PPP) approximation for the full many-electron $\pi$-shell Hamiltonian. For further formal and algorithmic details we refer to Appendix A. It is important that the SF-CIS computations can be easily made with the direct use of atomic orbital (AO) matrices, and without constructing explicitly the configuration interaction Hamiltonian matrices.

As mentioned in the introduction, before applying SF-CIS to large polyradicals, we must first compare the SF-CIS and $\pi$-FCI results for small $\pi$-radicals. This is done here for some typical conjugated tetraradicals. Table 1 shows the "spin gap" (energy $\lambda[Q \rightarrow T]$ of the lowest quintet-triplet tran- sition). In this Table we designate by I the tetraallyl structure, by II the symmetric tetraradical $\mathrm{C}_{10} \mathrm{H}_{14}$, and by III the $1,3,6$,8naphthoquinotetramethane. The carbon backbones of these systems are displayed in the last column where atomic spin-density diagrams are presented. We see that the SF-CIS model satisfactorily estimates the quintet-triplet transition energy $\lambda[Q \rightarrow T]$ (recall that $\pi$-FCI is the exact theory in the adopted $\pi$-methodology). At the same time other approximations, such as UHF and EHF schemes, give unsatisfactory results. For instance, we find that at the EHF level, $\lambda[Q \rightarrow T]=0.54 \mathrm{eV}$ in II, and $\lambda[Q \rightarrow T]=$ $0.76 \mathrm{eV}$ in III. Notice that our ( $\pi$-FCI result for $\lambda[Q \rightarrow T]$ in II is very close to that computed in [14], where it is shown that the lowest triplet state of II lies $0.16 \mathrm{eV}$ higher than the ground state quintet.

The spin-density distributions shown in Table 1 also reflect interesting features of high-spin systems. In all spin-density images given in Tables, the brown color corresponds to the positive spin density values, and the green color to the negative ones. The spin distributions will be discussed in 
Table 2. The first five energies $\lambda_{j}$ (in eV) of spin excitations $S_{0} \rightarrow S_{0}-1$ and spin density distribution for the ground state in large polyradicals within SF-CIS. $N_{\mathrm{C}}$ is a number of carbon atoms, and $S_{0}$ is the ground state spin.

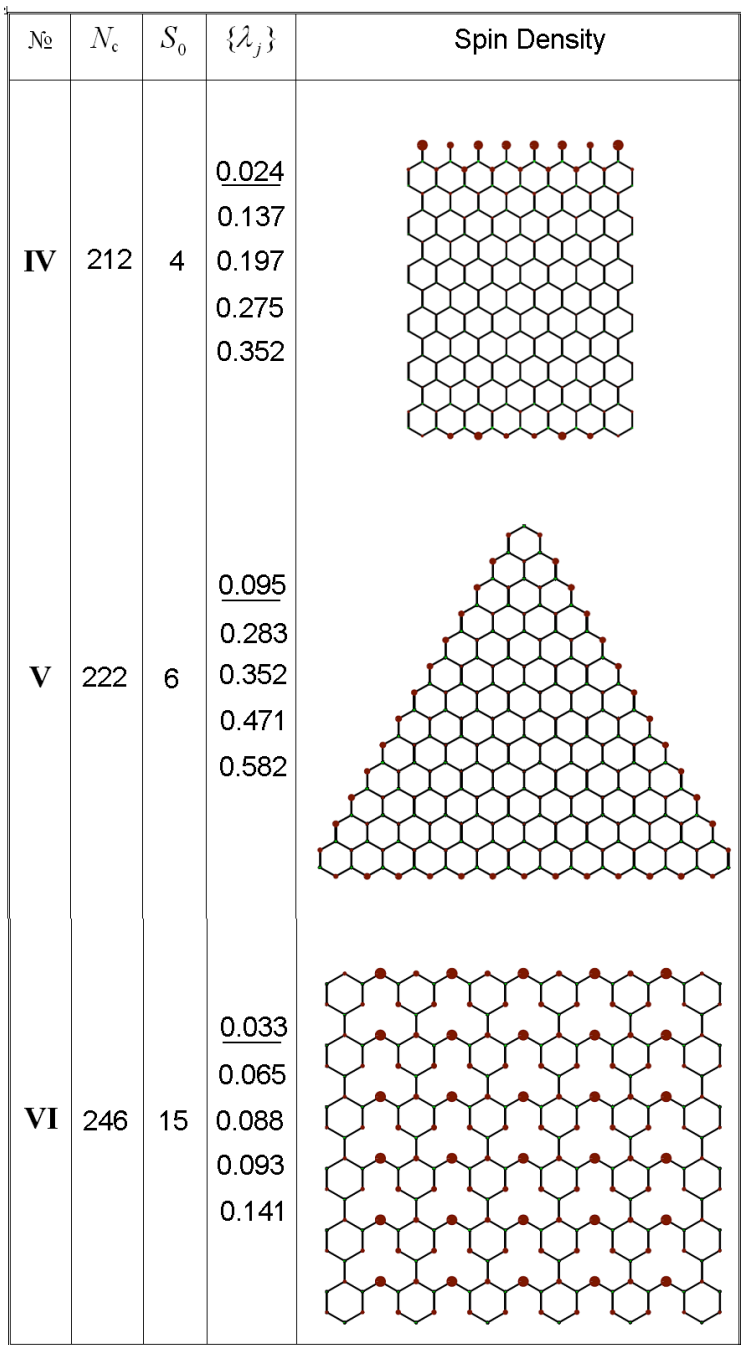

more detail later. We only mention that the SF-CIS atomic spin densities reasonably approximate the exact $(\pi-F C I)$ ones. In whole, the obtained results show the good performance of SF-CIS for small polyradicals, so we have a sufficient foundation for using this method in studying large polyradical systems.

\section{Large spin states in carbon-based clusters}

All the systems treated in this paper (except for Appendix C) are referred to as the so-called alternant hydrocarbons (the term was coined by Coulson and Rushbrooke [38]). More general is the graph-theoretic term "bipartite graphs" (e.g., see references in our recent paper [39]). For characterizing alternant hydrocarbons we must divide their carbon atoms into two disjoint sets (starred and unstarred) in such a way that no two atoms in the same set are directly linked. Let $m_{*}$ be the number of starred atoms and $m_{0}$ the number of unstarred atoms, and by definition $m_{*} \geq m_{0}$. Then the remarkable Lieb theorem [16] states that the quantity

$$
S_{0}=\left(m_{*}-m_{0}\right) / 2
$$

is the ground state spin of the bipartite lattice treated in the framework of the Hubbard many-electron theory (the counterpart of the $\pi$-FCI model with the one-center approximation to two-electron repulsion integrals). Ovchinnikov's rule [12] is of the same form since an infinite limit of onecenter repulsion integrals leads to the spinHamiltonian model used in [12]. The examples of tetraradicals from the preceding sec- 
tion, rule evidently, satisfy the LiebOvchinnikov (4).

After this preliminary, we consider typical nanographene molecules with the Kleintype edges $[40,41]$. The previously given system III in Table 1 is a simplistic example of the Klein-type edges in a small acene. In this section we study large polyaromatic structures of this type, that is the graphene nanoribbon with methylene edges and other polyradical structures (Table 2). Unlike [41] where only an indirect qualitative description (by non-bonding Huckel orbitals) is given for the related polyradical graphene ribbons, we base our study on approximate quantitative estimates of their spin excitation energies.

Let us now turn to Table 2 and see how SF-CIS works on the selected large polyradicals with different topology and spin. In the Table we give excitation energies $\lambda_{j}$ for spin transitions with lowering spin value, that is for $S_{0} \rightarrow S_{0}-1$. Spin-gap energies, i.e., energies of the lowest spin excitation, are underlined in the Table. The first example in Table 2 is the nanographene polyradical cluster IV. In this case, due to eight methylene groups and in accord with Eq. (4), $S_{0}=4$ is the spin of the ground state. It would be expected that the atomic spin densities would be predominantly localized on the corresponding $\mathrm{CH}_{2}$-decorated edge (at the top of the system). However, calculations show the well marked spin densities at the bottom as well. As a result of delocalization, the spin gap is very small in IV. The next interesting system is triangulene $\mathbf{V}$. Paper [12] was the first where the triangulene structures were proposed as organic magnets with large (microscopic) numbers of unpaired spins. The recent publication [42] provided a detailed account of the hard DFT computations of a sufficiently long series of such triangular molecules, but without giving spin excitation spectra. Our result concerning the spin density distribution are in concordance with those from [42]. As in the above cited work, the spin density is more preferably localized on the peripheral starred atoms, although the rest atoms slightly participate in the distribution as well. It is interesting that in the studied triangulene molecule the spin gap is about four times larger than in the system I having a comparable size and spin. Apparently, this peculiarity is a result of less symmetric structure in I. At last, let us consider the high-spin structure VI which gives a model of the Mataga-type polymer magnet from [11]. It is characterized by a small spin gap energy and significantly delocalized spins over all starred atoms of this system.

\section{Spin excitation spectra and heat capacity}

Here we analyze the spin excitation spectrum in the same polyradicals IV-VI. The first five excitation energies for them are given in the fourth column of Table 2 . We see that in the case of IV there is a sharp difference in the values of the first spin excitation energy (i.e. the spin gap) and the rest part of the spectrum. Such a difference is not so significant in the case of $\mathbf{V}$ and VI. This peculiarity is clearly reflected in the results for the magnetic contribution, $C_{\mathrm{v}}$, to the total specific heat capacity. The corresponding contribution can be easily calculated using standard expressions from statistical mechanics, namely

$$
C_{v}=\left(<\lambda^{2}>-<\lambda>^{2}\right) / T^{2} .
$$

Here $T$ is the absolute temperature (in $\mathrm{K}$ ), and $\langle\lambda\rangle$ and $\left\langle\lambda^{2}\right\rangle$ are found by averaging excitation energies $\left\{\lambda_{i}\right\}$ (measured in $\mathrm{K}$ ) and $\left\{\lambda^{2}{ }_{i}\right.$ over the canonical Gibbs distribution. When computing $C_{v}$ for spin systems, one must take explicitly into account level degeneracy (in our case, $2 s+1$ for the ground state, and $2 s-1$ for all excited states). We must also not forget that this specific heat is the magnetic contribution alone, which is superimposed on the vibrational heat capacity.

The results of computing $C_{v}$ from Eq. (5) are shown in Fig. 1. We see that the temperature dependence of $C_{v}$ in IV gives a very asymmetric two-humped curve, and indeed this behavior is essentially different from that of the other two systems. This peculiarity can be easily explained. To this end, let us first consider a two-level system allowing only the ground state and single excited state with the given excitation energy $\lambda_{i}=\lambda_{0}$ $\left(\lambda_{0}>0\right)$. Then it is easy to find that [43]

$$
C_{v}=e^{\zeta}\left(\frac{\zeta}{1+e^{\zeta}}\right)^{2} \text {, }
$$

where $\zeta=\lambda_{0} / T$. Its plot is displayed in the first panel of Fig. 2. We see that $C_{v}$ has a single maximum point $\left(C_{v} \cong 0.439\right.$ at $\lambda \cong$ 0.417 ), and this type of the $C_{v}$ temperature dependence is called the Schottky anomaly (the Schottky peak) [43,44]. The situation 


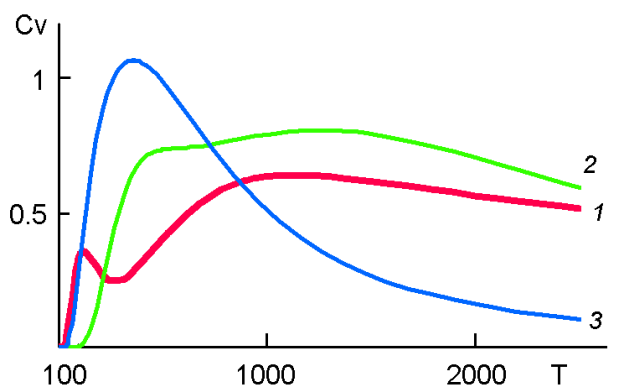

Fig. 1. Magnetic contribution to heat capacity $C_{v}$ of polyradicals IV (the red-color (1) line), $\mathbf{V}$ (the green-color (2) line) and VI (blue-color (3) line) versus temperature (in $\mathrm{K}$ ).

becomes more complicated when new energy levels are involved.

Thus, we next consider a simplest threelevel model. In it, in addition to the first excited state with excitation energy $\lambda_{0}$, we include the second (doubly-degenerate) excited level with excitation energy $k \lambda_{0}$, where coefficient $k \geq 1$. After manipulations over Eq. (5) we arrive at the following expression:

$$
\begin{gathered}
C_{v}= \\
=(\zeta / Z)^{2}\left(e^{-\zeta}+2(k-1)^{2} e^{-(k+1) \zeta}+2 k^{2} e^{-k \zeta}\right),
\end{gathered}
$$

where $Z=1+e^{-\zeta}+2 e^{-k \zeta}$. A plot of this function for $k=5$ is shown in the second panel of Fig. 2. Notice that for $k<4$ we find no qualitative difference with the above given case of the single Schottky peak. In the our three-level model, a second more broad peak starts its appearance when $k>4$. Returning to Fig. 2 we see that the second plot of this figure is just very similar to that given in Fig. 1 for IV (the red-color line). Then, a significant gap between the first excitation and the remaining part of the excitation spectrum (as in IV) can be a main cause for the observed two-humped peak anomalies in the temperature behavior of heat capacity.

It is also sensible to consider an elementary many-level model with equidistant spectrum. Unlike the harmonic oscillator, the system in study is assumed to have a finite number of energy levels. Thus, the excitation spectrum is taken as $\lambda_{j}=j \lambda_{0}$, where $1 \leq j \leq j_{\max }$, with $j_{\max }$ being a number of the excited states involved. The statistical sum and $\langle\lambda\rangle$ for this problem are trivially evaluated, but the end result for $C_{v}$ is a cumbersome expression, and here is not given explicitly. For a specific case when $j_{\max }=40$, the resulting $C_{v}$ plot is displayed in the third panel of Fig. 2. It is interesting that this plot is rather like the $C_{v}$ curve for polyradical $\mathbf{V}$ in Fig. 1 (the green-color line). As to possible causes for differences in spectra of IV-VI, we can give only a presumable explanation. Really, the considered systems have a comparable amount of carbon atoms, and they all are $\pi$-conjugated polyradicals. With this, spin value $S_{0}$ of the ground state does not correlate with the spin gap energy. Judging from our results in Table 1 and 2, the minimum spin gap is obtained when unpaired spins (they are localized on starred atoms) are situated as close as possible to each other (as in I and IV). However, this suggestion needs checking with more examples.

\section{Concluding remarks}

In this paper we considered molecular high-spin model magnets which are of interest for their possible application to microelectronics and nanoscience. By using the known SF-CIS technique, we could examine polyradicals having $\pi$-conjugated system on
Single excited state

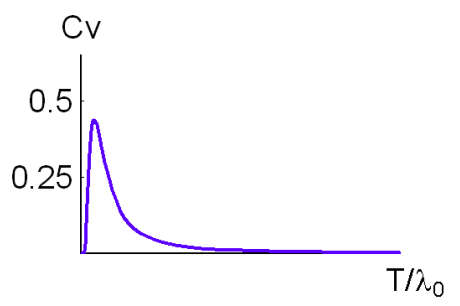

$\left(\lambda_{1}=\lambda_{0}\right)$
Two excited states

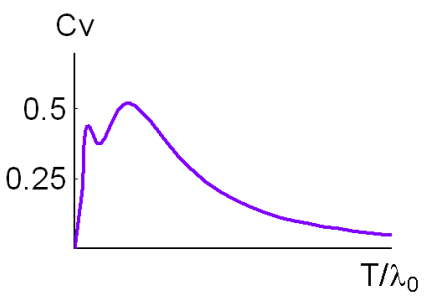

$\left(\lambda_{1}=\lambda_{0}, \lambda_{2}=5 \lambda\right)$
40 excited states

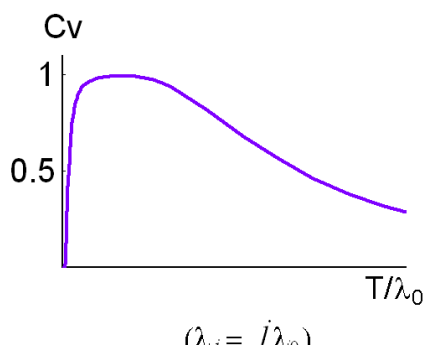

Fig. 2. Heat capacity $C_{v}$ for twolevel (the left panel), three-level (the middle panel), and multi-level (the right panel) models vs scaled temperature $T / \lambda_{0}$. 
the their backbone. It was found that the investigated systems demonstrate various types of spin excitation spectrum, and these variations can manifest itself in thermodynamic properties of these molecular magnets.

There are some additional interesting issues which we leave for further study. One is an extension of the SF-CIS technique to very large polyradicals when the appropriate polymeric polyradical can be cast as a translation-invariant problem. For this problem we must produce a spin-flip counterpart of the $\pi$-electron exciton model from [45], and modify the existing program codes described in [45, 46].

Another concerns the magnetic behavior of charged high-spin systems. In this case we cannot directly use SF-CIS for $\pi$-electron polyradicals with bipartite graph structure. Indeed, within $\pi$-electron theory, the charged states of these polyradicals involve degenerate open-shell reference wave functions, for which the conventional SF-CIS method is not applicable. However, there are special approaches (SF-CIS-2h,p from [36] and others) which allows us to obviate the problem. It seems that studying spin-excitation spectrum in charged molecular magnets will lead to intriguing magnetic effects.

At last, there are similar problems of predicting magnetic properties of carbonbased nanostructures with saturated bonds. For instance, it was observed that ion irradiation of nanosized diamond particles (nanodiamonds) with $\mathrm{N}$ and $\mathrm{C}$ shows a signature of ferromagnetism [47]. The spin cluster structures can be also produced in diamonds with a higher concentration of color nitrogen-vacancy (NV) centers [48]. Such defect-induced magnetism is another promising area for future research; see also the interesting review paper [50] where authors shortly discuss the magnetism of surface-induced localized spins in nanodiamonds (from $\sigma$-dangling bonds). In Appendix C, using the same SF-CIS technique as in above, we report our preliminary results of the study of NV centers in the model nanodiamond system.

Acknowledgement. The author thanks Dr.O.A.Zhikol for useful comments and Dr.I.V.Omelchenko for the collaboration in numerical computations of nanodiamond defects. The research was supported in part by the joint project of the National Academy of Sciences of Ukraine and the National Academy of Sciences of Belarus (Grant No.09-06-15).

\section{Appendix A: Working matrix equation}

Here we sketch the basic equations of the SF-CIS approach and give some additional useful details. Following Ref. [36], we first write down the variational SF-CIS equation for spin-flip amplitude matrix $t$. The equation takes the compact form:

$$
\left(I-\rho_{\beta}^{*}\right)\left[f_{\beta}^{*} t-t f_{\alpha}^{*}-K(t)\right] \rho_{\alpha}^{*}=\lambda \downarrow t .
$$

Here $\rho_{\alpha}{ }^{*}$ and $\rho_{\beta}{ }^{*}$ are the symmetric idempotent matrices projecting onto subspaces of spin-up and spin-down reference orbitals, respectively; matrices $f_{\alpha}{ }^{*}$ and $f_{\beta}{ }^{*}$ are the corresponding Fock matrices, and $K(t)$ the standard Roothaan exchange operator matrix. In this equation, $\lambda^{\downarrow}$ is an eigenvalue, and it has a meaning of a spin-flip energy.

The full set $\left\{\lambda_{j}{ }^{\downarrow}\right\}$ determines a branch of $S_{0} \rightarrow S_{0}-1$ transitions in the full spin excitation spectrum. Recall that the spin-flip procedure in Eq. (2) generates as well states with the same spin $S_{0}$ as in the reference determinant $\left|\Phi_{*}\right\rangle$, but these states have spin projection $S_{z}=S_{0}-1$. In ferromagnetic polyradicals, minimum spin-flip energy $\left\{\lambda_{\text {min }}{ }^{\downarrow}\right\}$ is just related to this type state with spin $S_{0}$. Therefore, the low-lying transition energies, $\lambda_{j}$, corresponding to lowering $\operatorname{spin}\left(S_{0} \rightarrow S_{0}-1\right)$ are obtained as differences $\lambda_{j}=\lambda_{j}{ }^{\downarrow}-\lambda_{\text {min }}{ }^{\downarrow}$. Possible problems with spin contamination are briefly touched upon in Appendix B. In passing, we mention a very recent paper [50] where SF-CIS and related spin-flip approaches are efficiently used for computing ab initio exchange integrals needed for the Heisenberg spin-Hamiltonian theory of molecular ferromagnets.

The main advantage of Eq. (A1) is that to solve it, we do not need using molecularorbital representation. Instead, one can directly work with matrices computed in $\mathrm{AO}$ representation. It is especially effective when dealing with semiempirical approximations. To be more specific, consider the conventional $\pi$-electron PPP scheme. The corresponding AO representation of $\rho_{\alpha}{ }^{*}$ and $\rho_{\beta}{ }^{*}$ and the Fock matrices $f_{\alpha}{ }^{*}$ and $f_{\beta}{ }^{*}$ are given in [51]. For instance, non-diagonal matrix elements of $f_{\alpha}^{*}$ are very easily computed to be

$$
\left(f_{\alpha}^{*}\right)_{\mu \nu}=\beta_{\mu \nu}-\left(\rho_{\alpha}^{*}\right)_{\mu \nu} \gamma_{\mu \nu},
$$

with $\beta_{\mu \nu}$ being the resonance integrals, and $\gamma_{\mu \nu}$ the two-center electron repulsion inte- 
gral for the given atomic $\pi$-centers $\mu$ and $v$. Thus, all matrices are of one-electron type, and it makes computations on Eq. (A1) much more easy than in respective ab initio (more rigorous) theories. Such efficiency becomes crucial when solving the eigenvalue problem (A1) by iterative methods of Krylov subspace type.

In our specific calculations, we take for $\beta_{\mu \nu}$ the usual nearest-neighbor approximation with the $\mathrm{C}-\mathrm{C}$ resonance integral $\beta_{\mathrm{C}}=$ $-2.4 \mathrm{eV}$; furthermore, integrals $\gamma_{\mu \nu}$ are computed by the standard Ohno formula with one-center repulsion integral $\gamma_{C}=11.13 \mathrm{eV}$. Molecular geometry was simplified to a regular honeycomb structure with $1.4 \AA$ for all $\mathrm{C}-\mathrm{C}$ bond lengths.

\section{Appendix B: Evaluation of spin density within SF-CIS}

In [36] we presented the working formula for the average total squared spin, $<S^{2}>$, of the SF-CIS (and more general SF) wave function. This formula (Eq. (52) in [36]) provides a correct identification of calculated states. For our purposes here the spin density matrix is important as well. The needed expression can be straightforwardly obtained by using a general relation [52] which expresses the one-electron spin density matrix, $Q$, in terms of spinless (charge) two-electron density.

The final result of our computations is

$$
Q=Q_{0}-t t^{+}-t^{+} t+\Delta Q
$$

where $Q_{0}=\rho_{\alpha}{ }^{*}-\rho_{\beta}{ }^{*}$, and $\Delta Q$ makes a correction depending of the $\left\langle S^{2}>\right.$ value. When $<S^{2}>=S_{*}\left(S_{*}+1\right)$ or around it, that is, the target state has the same spin as the reference determinant $\left|\Phi_{*}\right\rangle$, we have

$$
\Delta Q=\frac{\left(Q_{0}\right)^{2}-2 Q_{0}+3 t t^{+}+t^{+} t+L}{S_{*}+1},
$$

where

$$
\begin{aligned}
L=\left(t^{+} t\right) \rho_{\beta}^{*}+ & \rho_{\beta}^{*}\left(t^{+} t\right)-\left(t t^{+}\right) \rho_{\alpha}^{*}-\rho_{\alpha}^{*}\left(t t^{+}\right)+ \\
& +(\operatorname{Tr} t)\left(t+t^{+}\right) .
\end{aligned}
$$

When $<S^{2}>=S_{*}\left(S_{*}-1\right)$ or around it, that is, the target state has a lower spin $S_{*}-1$, the working formula for $\Delta Q$ is modified, as follows:

$$
\Delta Q=\left(Q_{0}\right)^{2}-Q_{0}+2 t t^{+}+L / S_{*},
$$

and Eq. (B3) remains untouched. From the above equation one can reproduce $<S^{2}>$ by tracing the $Q$-matrix in Eq. (B1):

$$
\begin{gathered}
<S^{2}>=\left(S_{*}-1\right)^{2}+ \\
+\operatorname{Tr}\left[\left(Q_{0}\right)^{2} / 2+\left(t^{+} t\right) \rho_{\beta}^{*}-\left(t t^{+}\right) \rho_{\alpha}^{*}\right]+|\operatorname{Tr} t|^{2} .
\end{gathered}
$$

This is just Eq. (52) Ref. [36]. In practical computations, as usual the low-lying SFCIS states have a correct value of $\left\langle S^{2}\right\rangle$ or the value near it. However, the spin contamination of SF-CIS states is typically large for higher excited states. For the latter, the spin projected SF-CIS methods should be used instead (see [36] for more detail).

\section{Appendix C: SF-CIS for triplet states of $N V$ color centers in nanodiamond}

Before starting we would like to mention recent papers and reviews on nanodiamonds and NV color centers [53-59]. In case of nanodiamonds and diamondoid molecules (condensed adamantanes), there are certain difficulties in obtaining reliable spectral data by conventional methods of quantum chemistry (e.g., see [54, 59]). In particular, we have sufficient reasons to believe that in nanodiamond molecules we should not be able to accurately predict electronic transitions without accounting for vibronic effects [54, 56]. That is why, in practice, even simple and rough models can be in use for restrictive semi-quantitative description of large, nanosized clusters. Our aim in this Appendix is to examine one very simple model of $\mathrm{NV}$ defects in nanodiamonds.

In the proposed model we start with the standard idealized geometry (as in the pure diamond lattice) and take a certain finitesize carbon cluster terminated by hydrogen atoms. In our computations below we used the cluster $\mathrm{C}_{100} \mathrm{H}_{72}$ of the $D_{3 d}$ symmetry. To produce the usual NV color center, we must replace one carbon atom by the nitrogen atom, and, additionally, make a vacancy at the nearest-neighbor site. If we restrict ourselves by the charged $\mathrm{NV}^{-}$defect, then the target states will be the ground triplet state, $T_{1}$, and the first excited state, $T_{2}$. The problem is how to compute such manyelectron states in a non-sophisticated fashion. Here, we invoked a slightly simplified CNDO (complete neglect of differential overlap) scheme. The CNDO semiempirical parameters were taken from [60] (except for CNDO resonance parameter $\beta_{C}^{0}$, for which we 
take $-16 \mathrm{eV})$. This parametrization is quite suitable for saturated hydrocarbons and diamondoids, in particular. The corresponding CIS estimations made for example of adamantane $\mathrm{C}_{10} \mathrm{H}_{16}$, give us the lowest excitation energy, $\lambda_{\text {min }}$, equal to $7.33 \mathrm{eV}$ (compare this with the most reliable theoretical estimation $\lambda_{\text {min }}=7.36 \div 7.49 \mathrm{eV}$ in [54]). In case of the color center, we made a further simplification by ignoring difference between resonance integrals of $\mathrm{C}-\mathrm{N}$ and $\mathrm{C}-\mathrm{C}$ bonds. A similar simplification was made for one-center electron repulsion integrals, i.e., $\gamma_{N}=\gamma_{C}$ in the standard notation. With this, the orbital electronegativity parameters for nitrogen were assigned the values of 1.25 times larger than those of carbon.

In order to obtain the $\mathrm{NV}^{-}$lowest triplet states we employed the SF-CIS procedure based on the ROHF quintet reference state (see Section 2). The obtained results are displayed in Table 3 . The main conclusion from the computations is that, in the model used, the finite-size cluster with center is of a triplet nature, as it should be [59]. Furthermore, a rather strong absorption from this ground state to the first excited triplet state is predicted by this model, and it is also in agreements with experiment.

In our computations, the lowest excited triplet state is found to be, in fact, quasidegenerate. The average value of the two lowest excitation energies from Table 3 (i.e., $2.44 \mathrm{eV}$ ) approximates well the experimental value of $2.2 \mathrm{eV}$ for the ${ }^{3} A_{2} \rightarrow{ }^{3} E$ vertical excitation energy observed for the diamond NV centre [53]. Notice that we used an asymmetric finite-size cluster model of the NV center, so the model does not possess the required trigonal symmetry as the single defect in real diamond crystals does. Rather, our asymmetric model is more relevant to the case of subsurface defect in nanodiamond.

Overall, the model describes main features of the $\mathrm{NV}^{-}$-center more or less satisfactorily. In particular, the three nearestneighbor carbon atoms to the vacancy bear the majority of the spin density (see Table 3). These atoms are shown in the image by green color. At the same time, there is no spin density on the nitrogen atom (blue color ball in the image). This is in concordance with EPR experiments and other theories [55, 57].

The same type model gives reasonable results in the case of the neutral color center $\mathrm{NV}^{0}$ for which we found the estimate $\lambda\left[{ }^{2} E \rightarrow{ }^{4} A_{2}\right]=0.66$ versus another theoreti-
Table 3. Lowest triplet-singlet, $\lambda\left[T_{1} \rightarrow S_{j}\right]$, and triplet-triplet, $\lambda\left[T_{1} \rightarrow T_{j}\right]$, transition energies (in eV) for color center modeled by nanodiamond cluster $\left[\mathrm{C}_{98} \mathrm{NH}_{72}\right]^{-}$, and atomic spin density distribution in the ground $\left(I_{1}\right)$ state of the cluster. Oscillator strengths are given in parentheses.

\begin{tabular}{|l|l|l|}
\hline$\lambda\left[\mathrm{T}_{1} \rightarrow S_{j}\right]$ & $\lambda\left[\mathrm{T}_{1} \rightarrow T_{j}\right]$ & Spin Density \\
\hline & & \\
& & \\
0.70 & $2.42(0.12)$ \\
0.81 & $2.46(0.06)$ \\
2.27 & $2.72(0.25)$ & \\
& &
\end{tabular}

cal values of 0.86 and 0.68 from [61] and [55], respectively. Obviously, the above CNDO-like approximation allows for calculations to be carried out on sufficiently large nanodiamond structures whose size prevents the application of high-level quantum chemistry methods.

\section{References}

1. $\pi$-Electron Magnetism: from Molecules to Magnetic Materials, ed. by J.Veciana, Springer, Berlin (2001).

2. Molecular Nanomagnets, ed. by D.Gatteschi, R.Sessoli, J.Villain, Oxford University Press, Oxford (2006).

3. S.N.Datta, C.O.Trindle, F.Illas, Theoretical and Computational Aspects of Magnetic Organic Molecules, Imperial College Press, London (2013).

4. Molecular Magnets, Physics and Applications, ed. by J.Bartolome, F.Luis, J.F.Fernandez, Springer, Heidelberg (2014).

5. B.Pilawa, Ann. Phys. (Leipzig), 8, 191 (1999).

6. M.Mitani, Y.Takano,Y.Yoshioka, K.Yamaguchi, J.Chem. Phys., 111, 1309 (1999).

7. J.A.Crayston, J.N.Devine, J.C.Walton, Tetrahedron, 56, 7829 (2000).

8. Magnetism: Molecules to Materials, vol.V, ed. by J.S.Miller, M.Drillon, Wiley, Weinheim (2005).

9. A.Rajca, Chem.Rev., 94, 871(194); A.Rajca, Adv.Phys. Org. Chem., 40, 153 (2005); N.M.Gallagher, A.Olankitwanit, A.Rajca, J. Org. Chem., 80, 1291 (2015).

10. H.C.Longuet-Higgins, J.Chem.Phys., 18, 265 (1950).

11. N.Mataga, Theor.Chim.Acta, 10, 372 (1968).

12. A.A.Ovchinnikov, Theor.Chim.Acta, 47, 297 (1978). 
13. J.N.Murrell, A.Hinchcliffe, Mol.Phys., 11, 101 (1966).

14. J.Koutecky, D.Dohnert, P.E.S.Wormer et al., J.Chem. Phys., 80, 2244 (1984).

15. A.I.Krylov, Acc.Chem. Res., 39, 83 (2006).

16. E.H.Lieb, Phys. Rev. Lett., 62, 1201 (1989).

17. K.Kusakabe, M.Maruyama, Phys.Rev.B, 67, 092406 (2003).

18. S.Bhowmick, V.B.Shenoy, J.Chem. Phys., 128, 244717 (2008).

19. W.L.Wang, S.Meng, E.Kaxiras, Nano Letters, 8, 241 (2008).

20. N.Ota, N.Gorjizadeh, Y.Kawazoe, J.Magn. Soc.Jpn., 34, 573 (2010).

21. M.Hatanaka, J.Phys.Chem.C, 116, 20109 (2012).

22. J.Kang, F.Wu, S.-S.Li et al., Appl.Phys.Lett., 100, 153102 (2012).

23. J.Li, A.Tang, Chem. Phys. Lett., 170, 359 (1990).

24. S.Li, J.Ma, Y.Jiang, J.Phys.Chem.A, 101, 5587 (1997).

25. M.M.Mestechkin, G.E.Whyman, Mol.Phys., 69, 775 (1990).

26. A.V.Luzanov, Theor.Exp.Chem., 17, 227 (1981); Teor. Eksp. Khim., 27, 413 (1991).

27. H.Bethe, Zeitschrift fur Physik, 71, 205 (1931).

28. D.C.Mattis, The Theory of Magnetism, Harper \& Row, New York (1965).

29. Y.Nagaoka, Phys. Rev., 147, 392 (1966).

30. G.-s.Tian, J.Phys.A: Math.Gen., 23, 2231 (1990).

31. M.Kollar, R.Strack, D.Vollhardt, Phys.Rev.B, 53, 9225 (1996).

32. A.V.Luzanov, Yu.F.Pedash, Theor.Exp.Chem., 18, 1 (1982); J.Struct.Chem., 23, 290 (1982).

33. A.I.Krylov, Chem. Phys. Lett., $\quad 338, \quad 375$ (2001).

34. D.Casanova, L.V.Slipchenko, A.I.Krylov, M.Head-Gordon, J.Chem.Phys., 130, 044103 (2009).

35. D.Casanova, M.Head-Gordon, Phys., Chem. Chem. Phys., 11, 9779 (2009).

36. A.V.Luzanov, J.Struct. Chem., 44, 729 (2004).

37. A.V.Luzanov, J.Quantum Chem., 108, 671 (2008).

38. C.A.Coulson, G.S.Rushbrooke, Proc.Cambridge Phil.Soc., 36, 139 (1940).
39. A.V.Luzanov, Functional Materials, 21, 437 (2014).

40. D.J.Klein, Chem. Phys. Lett., 217, 261 (1994); D.J.Klein, L.Bytautas, J.Phys.Chem.A, 103, 5196 (1999).

41. M.Hatanaka, in: Graphene Simulation, ed. by J.Gong, Intech, Rijeka, Croatia (2011), p.101.

42. M.R.Philpott, F.Cimpoesu, Y.Kawazoe, Chem. Phys., 354, 1 (2008).

42. C.Kittel, Thermal Physics, Wiley, New York (1969).

44. R.L.Carlin, Magnetochemistry, Springer, Berlin (1986).

45. A.V.Luzanov, J. Struct Chem., 43, 711 (2002).

46. P.Sony, A.Shukla, Comp.Phys.Comm., 181, 821 (2010).

47. S.Talapatra, P.Ganeshan, T.Kim et al., Phys. Rev. Lett., 95, 097201 (2005).

48. L.M.Pham, D.Le Sage, P.L.Stanwix et al., New J.Phys., 13, 045021 (2011).

49. T.Enoki, K.Takai, V.Osipov et al., Chem. Asian J., 4, 796 (2009).

50. N.J.Mayhall, M.Head-Gordon, J.Phys.Chem. Lett., 6, 1982 (2015).

51. A.Brickstock, J.A.Pople, Trans.Farad.Soc., 50, 901 (1954).

52. G.E.Vaiman, A.V.Luzanov, M.M.Mestechkin, Theor. Math. Phys., 28, $634 \quad$ (1976); A.V.Luzanov, G.E.Whyman, Int.J.Quantum Chem., 20, 1179 (1981).

53. Y.Ma, M.Rohlfing, A.Gali, Phys.Rev.B, 81, 041204 (2010).

54. F.Marsusi, J.Sabbaghzadeh, N.D.Drummond, Phys. Rev. B, 84, 245315 (2011).

55. A.Ranjbar, M.Babamoradi, M.H.Saani et al., Phys. Rev. B, 84, 165212 (2011).

56. C.E.Patrick, F.Giustino, Nature Comm., 4, 20 (2013).

57. V.A.Pushkarchuk, S.Ya.Kilin, A.P.Nizovtsev et al., Optics Spectrosc., 108, 247 (2010); A.P.Nizovtsev, S.Ya.Kilin, A.L.Pushkarchuk et al., New J.Phys., 16, 083014 (2014).

58. C.Bosted, L.Landt, T.Moller et al., in: Nature's Nanostructures, ed. by A.Barnard, H.Guo, Pan Stanford Publishing, Singapore (2012), p.169.

59. M.W.Doherty. N.B.Manson, P.Delaney et al., Phys. Rep., 528, 1 (2013).

60. N.Ohmichi, A.Tajiri, T.Nakajima, Bull.Chem. Soc.Jpn., 45, 3026 (1972).

61. A.Gali, Phys. Rev.B, 79, 235210 (2009). 